\title{
Dynamic Analysis and FPGA Implementation of New Chaotic Neural Network and Optimization of Traveling Salesman Problem
}

\author{
Li Cui $\mathbb{D}^{1},{ }^{1}$ Chaoyang Chen, ${ }^{1}$ Jie Jin, ${ }^{1}$ and Fei Yu $\mathbb{D}^{2}$ \\ ${ }^{1}$ Hunan University of Science and Technology, Xiangtan, Hunan 411201, China \\ ${ }^{2}$ School of Computer and Communication Engineering, Changsha University of Science and Technology, Changsha 410114, China
}

Correspondence should be addressed to Li Cui; 348640501@qq.com

Received 19 February 2021; Revised 18 March 2021; Accepted 10 April 2021; Published 20 April 2021

Academic Editor: Chong Fu

Copyright (c) 2021 Li Cui et al. This is an open access article distributed under the Creative Commons Attribution License, which permits unrestricted use, distribution, and reproduction in any medium, provided the original work is properly cited.

A neural network is a model of the brain's cognitive process, with a highly interconnected multiprocessor architecture. The neural network has incredible potential, in the view of these artificial neural networks inherently having good learning capabilities and the ability to learn different input features. Based on this, this paper proposes a new chaotic neuron model and a new chaotic neural network $(\mathrm{CNN})$ model. It includes a linear matrix, a sine function, and a chaotic neural network composed of three chaotic neurons. One of the chaotic neurons is affected by the sine function. The network has rich chaotic dynamics and can produce multiscroll hidden chaotic attractors. This paper studied its dynamic behaviors, including bifurcation behavior, Lyapunov exponent, Poincaré surface of section, and basins of attraction. In the process of analyzing the bifurcation and the basins of attraction, it was found that the network demonstrated hidden bifurcation phenomena, and the relevant properties of the basins of attraction were obtained. Thereafter, a chaotic neural network was implemented by using FPGA, and the experiment proved that the theoretical analysis results and FPGA implementation were consistent with each other. Finally, an energy function was constructed to optimize the calculation based on the CNN in order to provide a new approach to solve the TSP problem.

\section{Introduction}

Undoubtedly, the human brain is the most complex and wonderful information processing organ. It was formed by humans after a long-term natural evolution and contains approximately 100 billion neurons. These neurons transmit information to each other to perform cognitive functions and control human behavior characteristics and thoughts. The brain is part of the central nervous system (CNS) in the structure of the human body, which is composed of a large number of neuronal cells and is connected by about $10^{15}$ synapses, thus forming a complex neural network that transmits information in an orderly and hierarchical manner. McCulloch and Pitts abstracted human brain neurons and built a simple model to form a neural network, namely, artificial neural network [1]. Artificial neural network can be divided into three categories: shallow perceptron, simple artificial neural network, and deep neural network [2-13].
The neuron is the basic processor of the neural network. Each neuron has an output, which generally relates to its state and may affect several other neurons. Each neuron receives some input from connections called synapses. The input is the activated input neuron multiplied by the neuron's synaptic weight. The activated neuron is calculated by applying a threshold function to the product, and the threshold function is modeled by a nonlinear function. When designing a neural network, the most important thing is to ensure that the dynamic system converges on the corresponding system. On the other hand, the richer the dynamics are, the wider the range of applications would be. For example, when a neural network model is used as an approximate method to solve combinatorial optimization problems, the transient chaotic nature provides higher search performance for global optimization or approximate optimization solutions. By considering the sum of time and space of external input and feedback input from chaotic neurons, a chaotic neural network can be constructed with 
chaotic neurons. The study in [14] proposed a new fourdimensional chaotic neural memory cell neural network, studied its dynamic behaviors, and designed chaotic synchronization based on sliding mode control. The proposed chaotic memory CNN system can be used for secure communication. The study in [15] studied the construction of a blind restoration model for a superresolution image based on a chaotic neural network. In the paper, a simplified chaotic neural network model is first constructed. The gray value of the image is used as the input of the network. The generated Toeplitz matrix is used to calculate the connection weight and bias input of the chaotic neural network. Hence, the problem that the traditional blind restoration model for superresolution image based on neural network falls into the local minimum is solved. The study in [16] considered the circuit implementation and application of chaotic neural networks of reconfigurable memory. The chaotic neural network has been widely applied in associative memory because of its rich chaotic characteristics. In the paper, not only was circuit implementation performed, but also the autoassociative memory, heteroassociative memory, superimposed pattern separation, many-to-many associative memory, and their application in three-view drawing were realized through simulation experiments. The study in [17] examined the local synchronization control of chaotic neural networks with saturated actuators and sample data. The author of [18] studied the global power rate synchronization of chaotic neural networks with proportional delays based on impulse control. The study in [19] analyzed the sliding mode synchronization control of time-delayed chaotic neural networks based on the observer. The study in [20] proposed a chaotic neural network for encryption. The study in [21] considered the dynamic behaviors of chaotic circuits in neural networks. The study in [22] inspected the chaotic multistability problem of neural networks based on memristors.

Inspired by previous work, we simulated and studied a chaotic neural network consisting of a linear matrix, a sine function, and three chaotic neurons, one of which is affected by the sine function. In this paper, we first propose a new chaotic neural network model, followed by performing a nonlinear dynamic analysis on it, including bifurcation behavior, Lyapunov exponential spectrum, Poincaré surface of section, and basins of attraction analysis, and give out the FPGA implementation of chaotic neural network. Fewer researches have been conducted in the existing chaotic neural network research literature; therefore, the research on dynamics of this type of system is of paramount importance and meaningful.

\section{Chaotic Neural Network Model}

In this paper, based on the Hopfield neural network model, we extend the external and internal membrane conductance of neurons to the linear layer of the neural network model. A new chaotic neural network model is proposed as shown in the following equation:

$$
C_{i} \dot{x}_{i}=\sum_{j=1}^{n} S_{i j} x_{i}^{T}+\sum_{j=1}^{n} w_{i j} v_{j}+i_{i},
$$

where $x_{i}$ is the voltage on the capacitor $\mathrm{C}_{i}, \mathrm{~S}_{i j}$ is the conductance of membrane resistance of the outside and inside neurons, $I_{i}$ is the nonlinear external input current, and the matrix $W=W_{i j}$ is the synaptic weight of the connection strength between neurons. The activation function of neuron $V_{j}$ is defined as

$$
v_{j}=\tanh \left(x_{i}\right),
$$

and when $C_{i}=1$ and $n=3$, the new chaotic neural network model is shown in the following equation:

$$
\dot{x}_{i}=\sum_{j=1}^{3} S_{i j} x_{i}^{T}+\sum_{j=1}^{3} w_{i j} v_{j}+i_{i},
$$

where $i_{i}=\left[i_{1}, i_{2}, i_{3}\right]^{T}=\left[0,0, i_{3}\right]^{T}$ and $i_{3}=A \sin \left(x_{1}\right)$.

$$
\begin{aligned}
& S_{i j}=\left[\begin{array}{ccc}
0 & 2 & 0 \\
0 & 0 & 1 \\
0 & -3 & -5
\end{array}\right], \\
& w_{i j}=\left[\begin{array}{ccc}
5 & 0 & -1 \\
-6 & 2 & 4 \\
-1 & -3 & 10
\end{array}\right],
\end{aligned}
$$

where $A=20$; equation (3) can be rewritten as follows:

$$
\left\{\begin{array}{l}
\dot{x}_{1}=2 x_{2}+5 \tanh \left(x_{1}\right)-\tanh \left(x_{3}\right), \\
\dot{x}_{2}=x_{3}-6 \tanh \left(x_{1}\right)+2 \tanh \left(x_{2}\right)+4 \tanh \left(x_{3}\right), \\
\dot{x}_{3}=-3 x_{2}-5 x_{3}-\tanh \left(x_{1}\right)-3 \tanh \left(x_{2}\right)+10 \tanh \left(x_{3}\right)+20 \sin \left(x_{1}\right),
\end{array}\right.
$$

Connection of the neural network with three neurons is shown in Figure 1.

The chaotic neural network proposed in this paper can be regarded as a nonlinear associative memory or contentaddressable memory, which functions to retrieve the pattern in the memory to respond to the incompleteness or noise presented by this pattern. The essence of content-addressable memory is to map a basic memory $x_{i}$ to a stable fixed point in a dynamic system, where the stable fixed point of the network phase space is the basic memory of the network. We can describe their specific pattern as the starting point of the phase space. If the starting point is close to the fixed point, it represents the memory to be retrieved, and the system should converge on the memory itself over time. Therefore, the chaotic neural network is a dynamic system, whose phase space contains a set of stable fixed points that represent the basic system memory.

In this paper, we use the fourth-order Runge-Kutta methods to solve system (4), set the initial value to $(0.1,0.1$, 0.1 ), and obtain the phase diagram of system (4). From the phase diagram, the system can produce a four-scroll chaotic attractor. For details, see Figures 2(a)-2(c).

However, we found that the proposed system (4) has infinitely many equilibrium points and the Lyapunov 


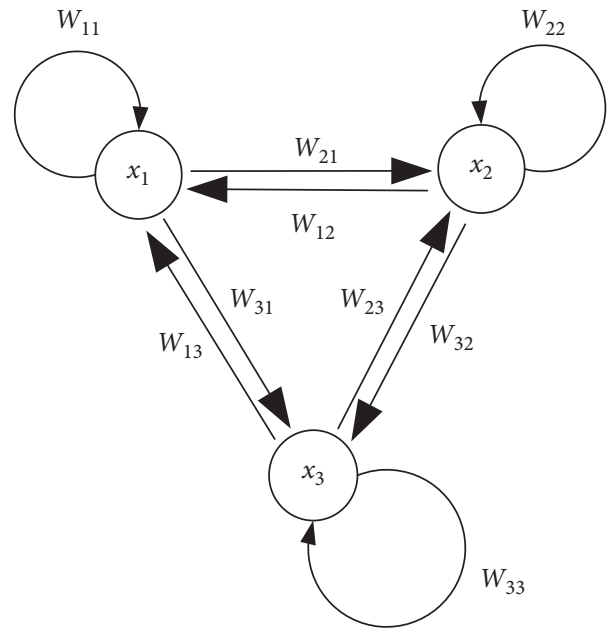

Figure 1: Connection of the neural network with three neurons.

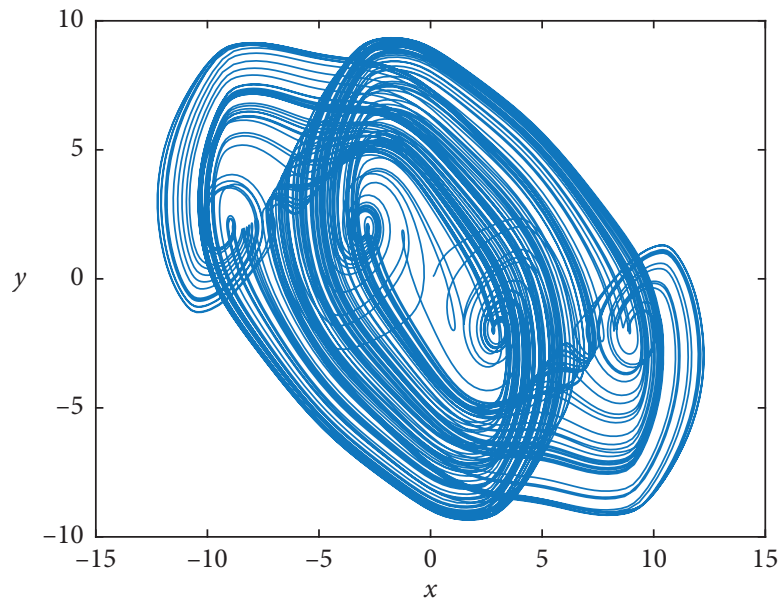

(a)

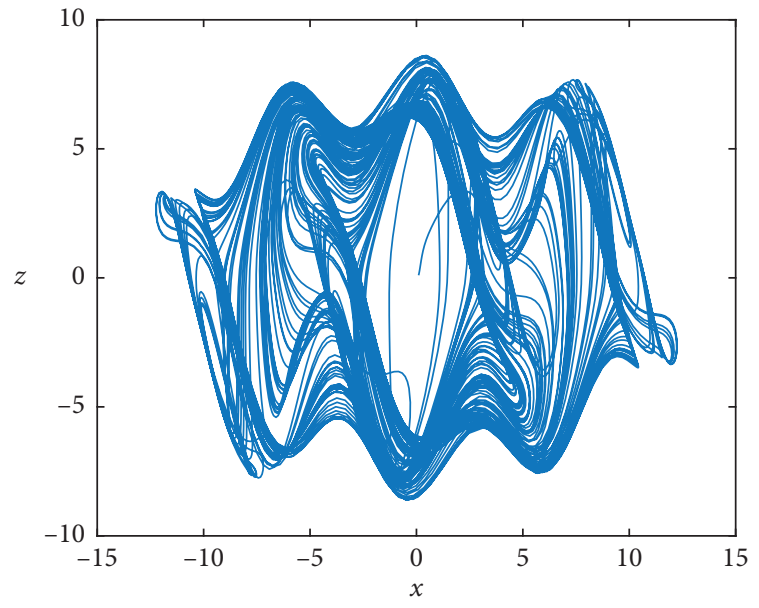

(b)

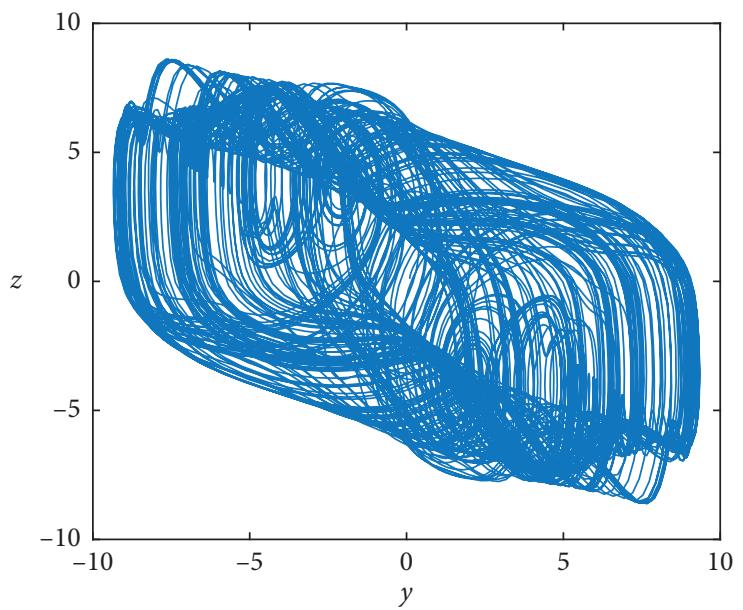

(c)

Figure 2: The chaotic attractor of system (4). (a) $x-y$ plane, (b) $y-z$ plane, and (c) $x-z$ plane. 
exponents of system $(4)$ are LE1 $=0.560261$, LE2 $=-0.001804$, and LE3 $=-4.056202$, so system (4) is a multiscroll hidden attractor system. The Lyapunov exponents are shown in Figure 3.

\section{Analysis of Bifurcation, Lyapunov Exponent, and Poincaré Section}

The control parameter A of system (4) changes from 0 to 22, and the initial value of system (4) is $(0.1,0.1,0.1)$. The step size of $\mathrm{A}$ is 0.04 , and the bifurcation diagram of $\mathrm{A}$ of system (4) is shown in Figure 4(a). System (4) bifurcates from period doubling into chaos over time.

We can observe the dark lines in the bifurcation diagram. It is generally believed that all solid lines disappear after the bifurcation point; however, they can still be solved by algebraic equations; therefore, there is no reason to stop after the bifurcation point. But why cannot we see it? This is because they have become unstable periodic orbits after bifurcation. Then why cannot the unstable periodic orbit be seen in the bifurcation diagram? Essentially, this is a question of how to track unstable periodic orbits. Because it is a hidden attraction subsystem, every point on the unstable periodic orbit is unstable. As long as there is a little error, it will deviate more and more from the equilibrium point. The hidden attraction subsystem itself is affected by the initial state. In the bifurcation diagram, we can also observe hidden bifurcation phenomena.

The most essential element about the positive Lyapunov exponent is the source of local instability of the chaotic attractor. One of the most basic characteristics of chaos is its high sensitivity to initial conditions. The two orbits produced by two very close and different initial values will separate exponentially over time, causing this kind of orbit to separate exponentially. The root cause of this phenomenon is the positive Lyapunov exponent in the chaotic system. Therefore, the Lyapunov exponent essentially describes the local instability in a chaotic motion. However, if there is only this local instability factor, the entire attractor will diverge, and, as a matter of fact, the chaotic attractor only exists in a certain range of phase space. Therefore, we believe that there should be multistability factors in the hidden chaotic attractor, in addition to the factor of local instability. The hidden chaotic attractor is the result of the interaction of two trends, namely, local instability and multistability, finally forming the fractal structure of the whole chaotic attractor. It fully reflects the fact that the hidden chaotic attractor is a dialectical unity of local instability and multistability. In the next section of this paper, we will use the Lyapunov exponent to describe the basins of attraction of system (4) [23, 24].

The analysis from Figure 4(b) shows how the positive Lyapunov exponent changes, which suggests that system (4) alternates between the quasiperiodic state and the chaotic state and fully depicts the changes in local instability and multistability of system (4). This is basically the same as the state change shown in the bifurcation diagram.

The continuous trajectory of the phase space appears as some discrete mapping points on the Poincaré section. If the transition process in the initial stage is ignored, only the steady-state image of the Poincaré section is considered. When there is only one fixed point and several discrete points on the Poincaré section, it can be determined that the motion is quasi-periodic. When the Poincare section presents a closed curve, it can be determined that the motion is quasi-periodic. When there is a dense point on the Poincaré section with a hierarchical structure, it can be determined that the motion is in the chaotic state.

The Poincaré map of the $x-z$ plane of system (4) is shown in Figure 5. The Poincaré diagrams of the system on different planes show many dense points, which shows that the system has chaotic bifurcation characteristics and folding ability.

\section{Analysis of Basins of Attraction}

For chaotic neural networks, we can analyze the stability of the system by considering the Lyapunov exponential function (energy function) of the system. When the network is operating in the initial state, the network will move in the direction in which the Lyapunov exponential function decreases until it reaches a local minimum. The local minimum point of the Lyapunov exponential function represents the stable point of the phase space. Each attractor is around a substantial basin of attraction. In this sense, these points are also called attractors. These basins of attraction represent a stable network state. When a stable point enters the lowest area of the basins of attraction, the solution in the network can be obtained. The size of the basins of attraction is described by the radius of attraction, which can be defined as the maximum distance between all states contained in the basins of attraction or the maximum distance at which an attractor can attract a state. The number of attractors represents the memory capacity or storage capacity of the associative memory network, while the storage capacity is the maximum number of noninterfering memories in the network within a certain tolerance of the associative error probability. The storage capacity is related to the allowable error of associative memory, network structure, learning method, and network design parameters. In short, the more attractors are present in the network, the greater the storage capacity is. The basins of attraction of the attractor act as an index to measure the fault tolerance of the network, that is, the larger the basins of attraction, the better the fault tolerance performance of the network, and the stronger the association ability of the network.

In a dynamic system with multiple attractors, the corresponding basins may have fractal boundaries and an even more complex structure. Therefore, this means that in system (4) a coexisting attractor will have such a complicated basins boundary structure. The red area represents the basins of attraction of the attractor at infinity, which is the point set where the trajectory diverges. The yellow area represents the basins of attraction of the chaotic attractor, which shows the coexistence of multiple attractors and the fault tolerance of the network. The larger the yellow area, the better the fault tolerance of the network. The blue area is the transition area. The section of the basins of attraction is a series of 


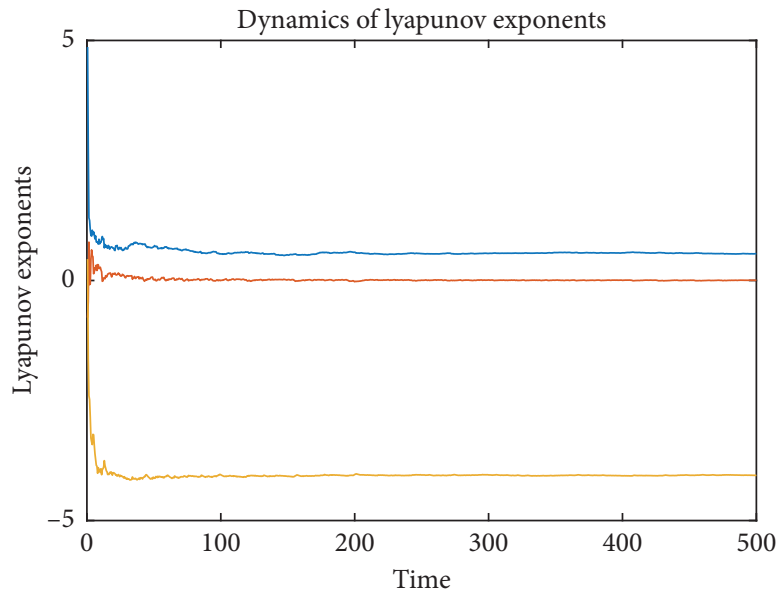

FIgURe 3: Lyapunov exponents of system (4).

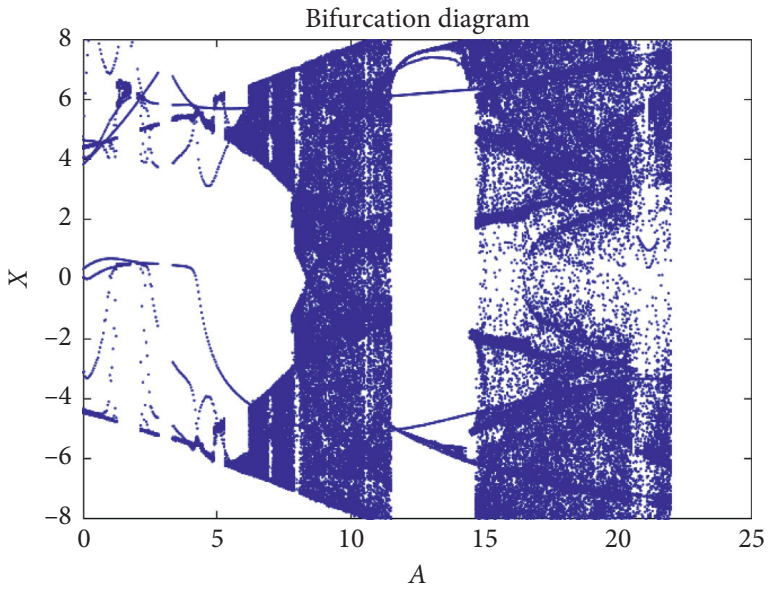

(a)

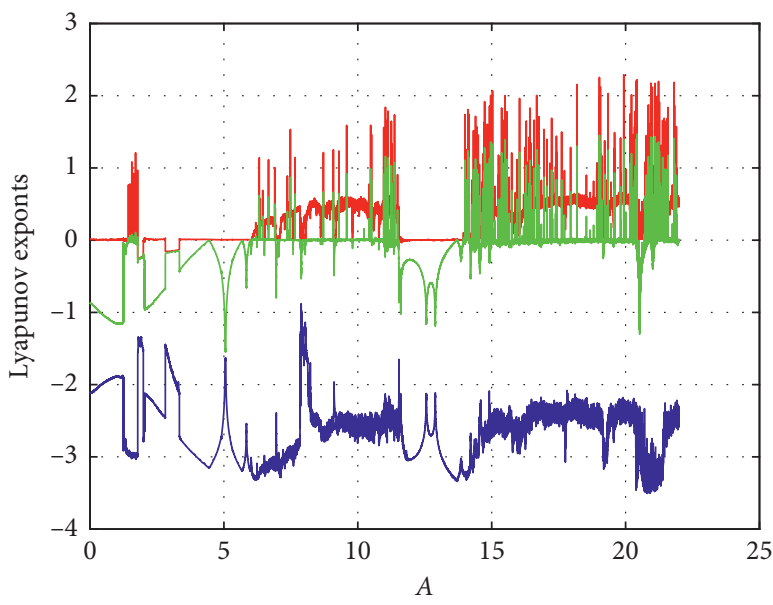

(b)

Figure 4: (a) The bifurcation diagram of A. (b) Spectrum of Lyapunov exponents changing A from 0 to 22.

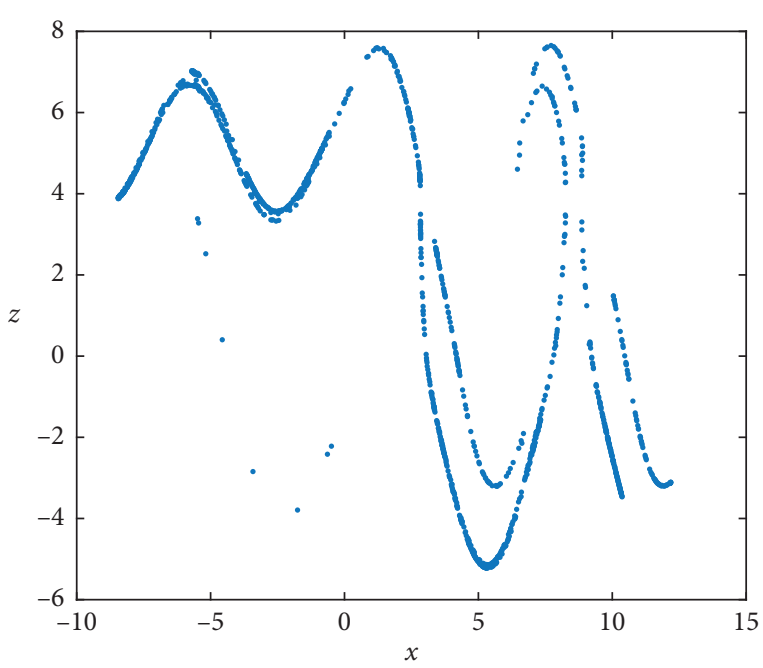

Figure 5: Poincaré map in the $x-z$ plane. symmetrical filaments, which are unevenly distributed but have a self-similar appearance. From Figure 6, it can be found that system (4) has four coexisting attractors.

The criteria for the existence of basins of attraction in a dynamic system [25] are as follows:

(1) There is a smooth invariant subspace containing chaotic attractors

(2) There is another asymptotic final state outside the invariant subspace (not necessarily a chaotic state)

(3) The lateral Lyapunov exponent of the invariant subspace is negative

(4) The lateral stability of the unstable periodic orbit of the attractor is related to the positive finite time change

The study in [25] has proved that the coupled Lorenz system satisfies conditions 1 and 2 . There are two invariant (three-dimensional) manifolds in the six-dimensional phase 


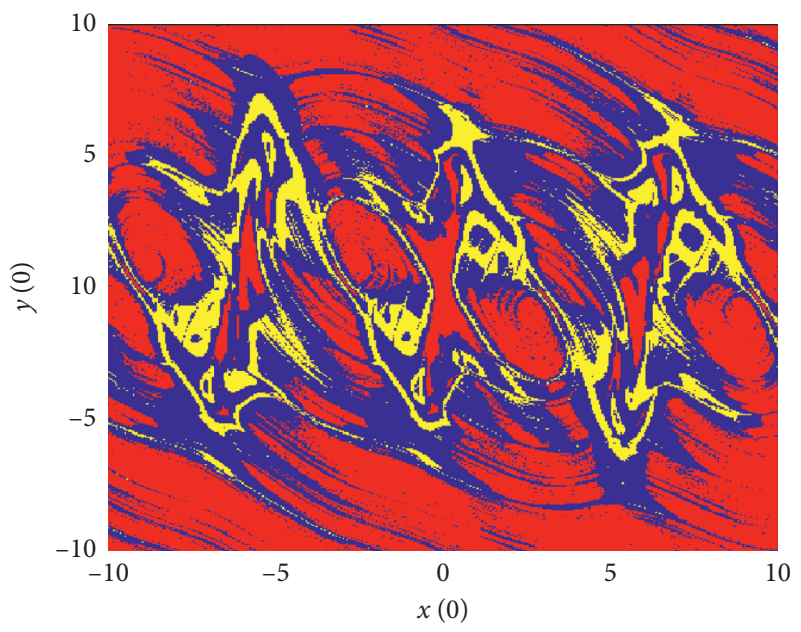

(a)

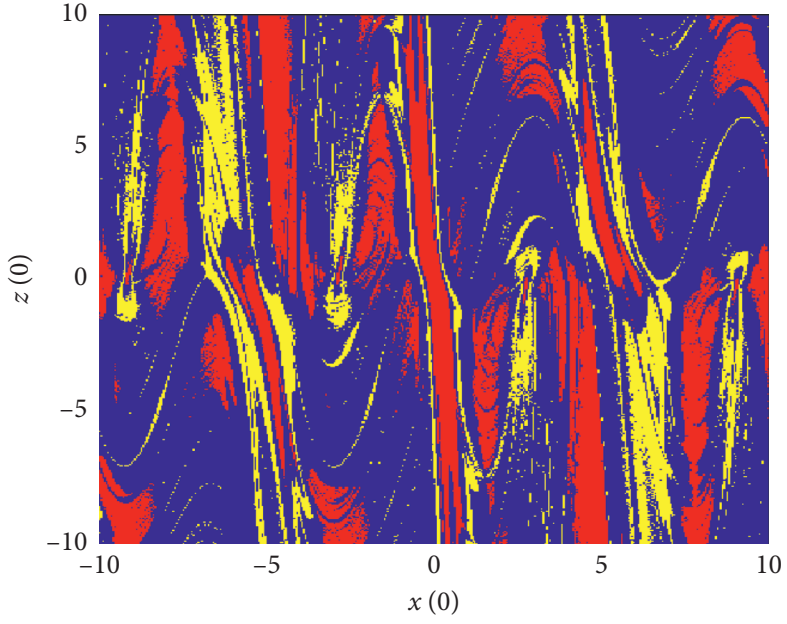

(b)

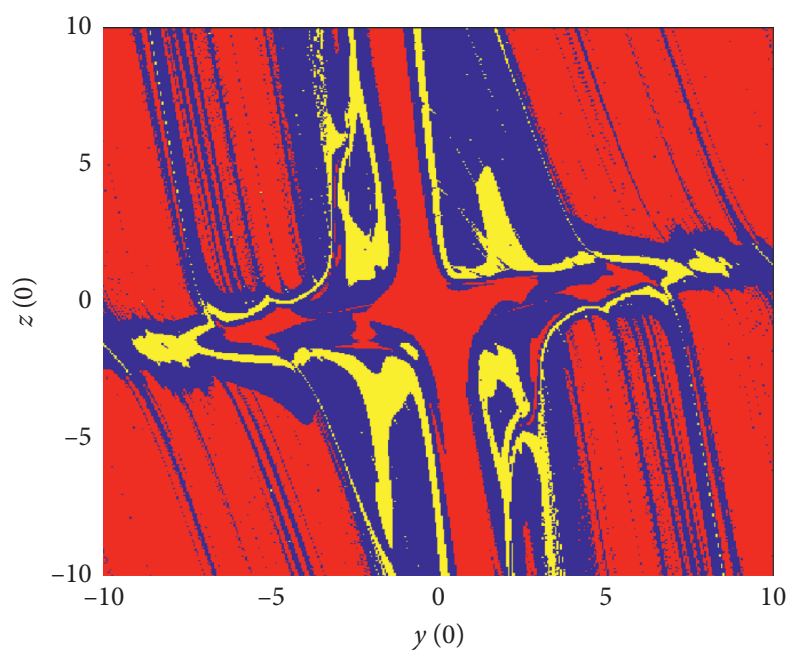

(c)

Figure 6: Basins of attraction of system (4). (a) $x(0)-y(0)$ plane, (b) $x(0)-z(0)$ plane, and (c) $y(0)-z(0)$ plane.

space; as the trajectory from each subspace will always remain there, it will evolve toward the respective famous Lorenz attractor. For the synchronous attractor of the coupled Lorenz system, the literature has proposed using the sieve area to describe the synchronous attractor of the coupled Lorenz system. In this paper, we use the finite-time lateral Lyapunov exponent for comparison with the lateral exponent of a specific orbit and obtain results similar to those in [25].

We find that the basins of attraction of the dynamic system have the following properties:

(1) The system orbit tends to a fixed point

(2) The system is periodic and quasi-periodic

(3) The system has chaotic or hyperchaotic behaviors

(4) The time series of the system tends to infinity in a finite time

The improvement of the associative memory network must overcome a fundamental problem; that is, in addition to the attractors with memory samples, there are also "redundant" stable states (pseudostates). The existence of pseudostates affects the fault tolerance of the associative memory network. If the basins of attraction of pseudostates can be reduced or eliminated, the fault tolerance of the associative memory network can be improved and the memory capacity can be increased.

\section{FPGA Implementation}

The hardware experiment of system (4) is conducted by the method of fixed-point number, based on FPGA technology. We use Xilinx Zynq-7000 series XC7Z020 FPGA chip and AN9767 dual-port parallel 14-bit digital-to-analog conversion module with the maximum conversion rate of $125 \mathrm{MHz}$ and adopt Vivado 17.4 and the System Generator to realize the joint debugging of Matlab-FPGA. Besides, we use oscilloscope to visualize the analog output. After the analysis, synthesis, and compilation of Vivado, to further confirm that the chaotic neural network system is correct, after confirming that the timing simulation results are correct, we generate the bit file by Vivado and download the generated 


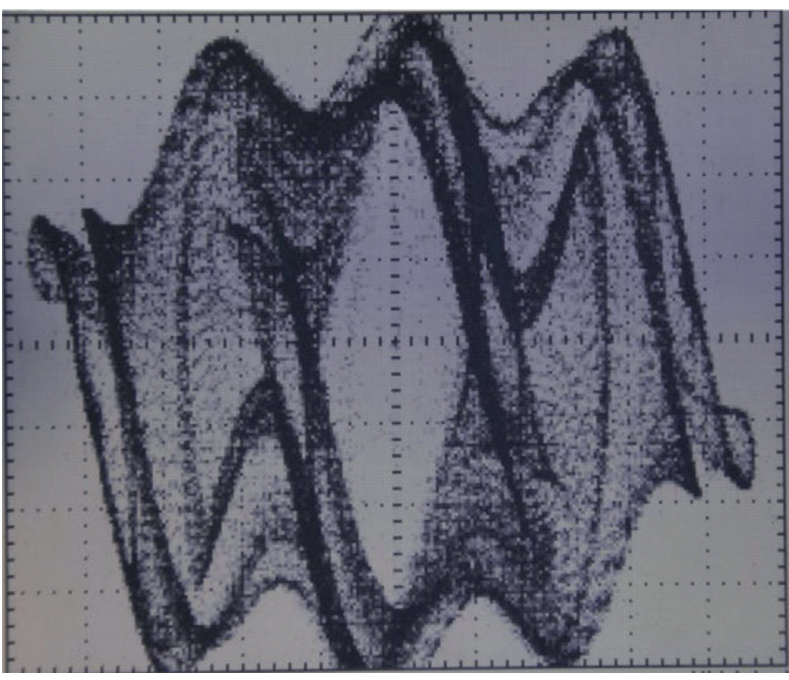

(a)

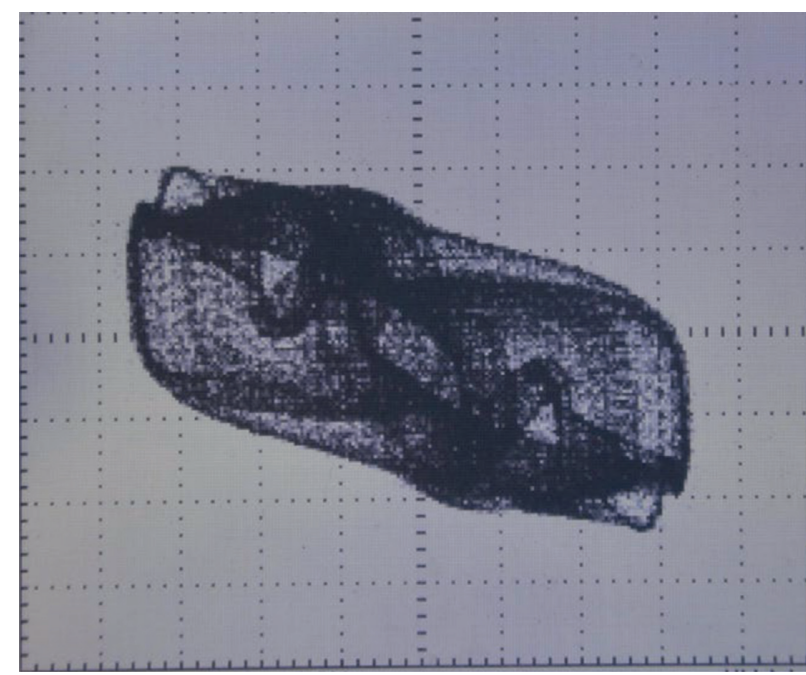

(b)

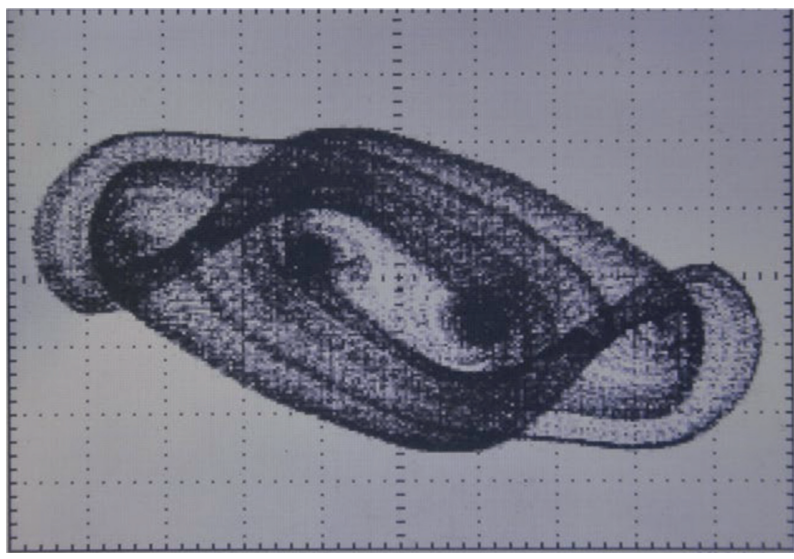

(c)

Figure 7: The chaotic attractor of system (4). (a) $x$-z plane, (b) $y$ - $z$ plane, and (c) $x-y$ plane.

bit file to the FPGA development board, convert the output of FPGA into the analog signal using AN9767 digital-toanalog converter, and then connect AN9767 digital-toanalog converter to the oscilloscope to observe the phase diagram of system (4) attractor. The phase diagrams displayed by the oscilloscope are, respectively, shown in Figure 7.

\section{CNN-Based Optimization Calculation of TSP Problem}

Traveling salesman problem (TSP) is a classic topic about combinatorial optimization. In a typical TSP scenario, a salesman has to rush around from one city to another to promote his goods and then goes back to his original city. How should he choose his shortest route through all the cities?

According to the Graph Theory, this problem is, in essence, to find out a Hamiltonian loop with the lowest weight in a weighted and directless graph. Given that the feasible solution to this problem is the total permutation of all the vertexes, as the vertexes increase, the resulting combinations explode; therefore, it is an NP-completed problem. The extensive application of this problem in transportation, circuit board, circuit design, and logistics distribution has led to its extensive research among scholars both at home and abroad. Various exact algorithms were used in early research to solve it, for example, branchand-bound technique, linear programming technique, and dynamic programming technique. However, as the problem snowballs in scale, these methods fail to work anymore. Therefore, in later research, scholars turn to approximate or heuristic algorithms, mainly including Genetic Algorithm $[26,27]$, Simulated Annealing [28, 29], Ant Colony Algorithm [30, 31], Tabu Search Algorithm [29, 32], Greedy Algorithms [33, 34], and neural networks [35].

The chaotic neural network (CNN) herein is a feedback neural network structured similarly to a control system where there is a feedback from an output terminal to the corresponding input one. Upon a given input excitation, the state of the loop will change continuously and the values at both the input and output terminals also do so until being stable. Each output represents a state. Therefore, the CNN herein is a power system with multiple inputs and outputs. In a dynamic system, the equilibrium state can be 


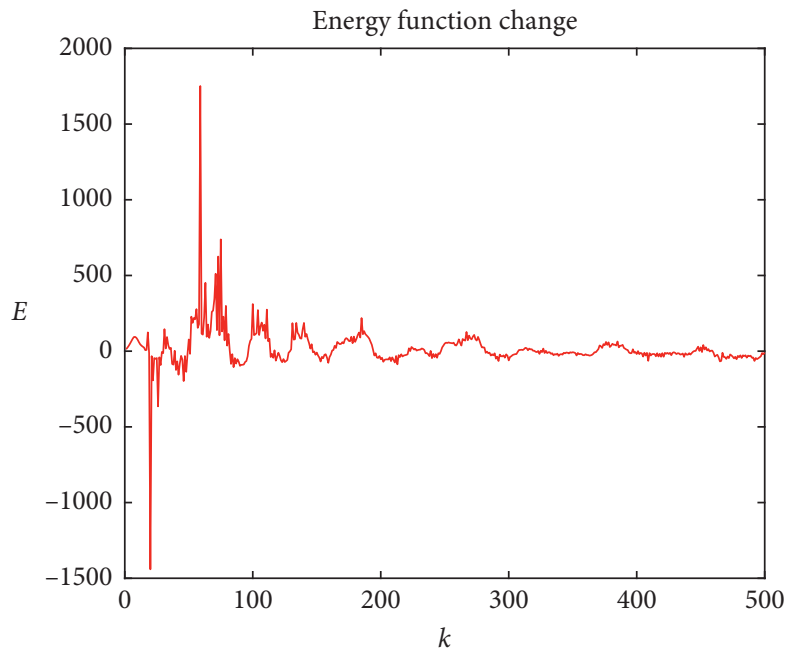

Figure 8: Change of energy function.

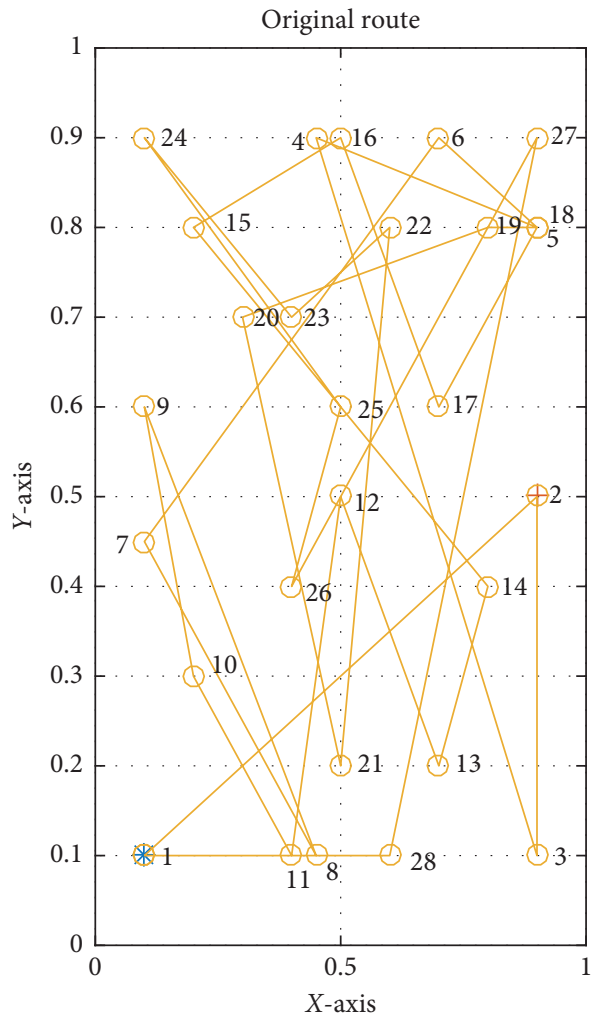

(a)

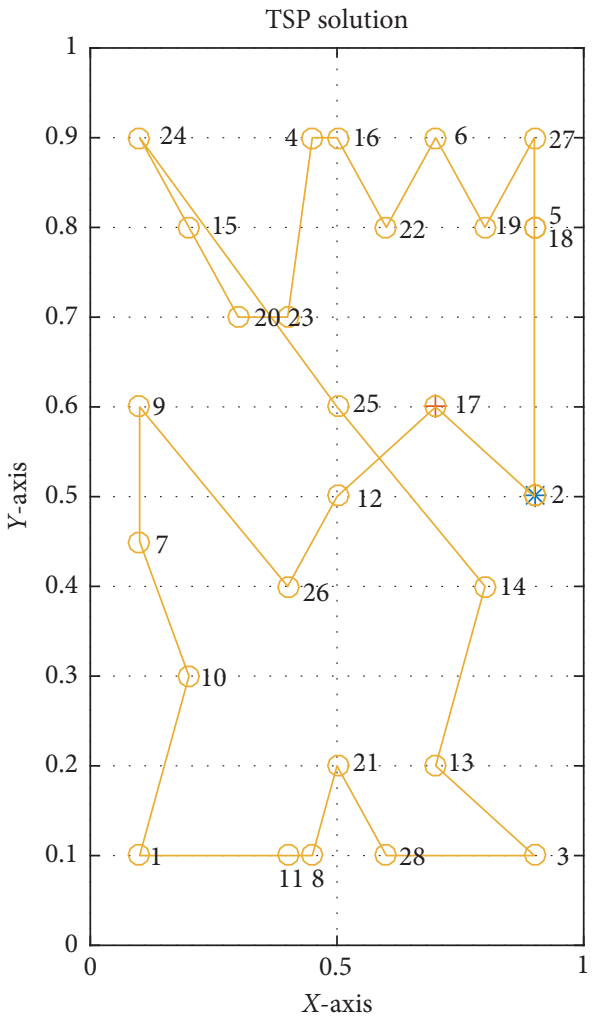

(b)

FIGURE 9: The solution of TSP

understood as the one in which the value of a form of energy in a dynamic system continuously decreases to the minimum. The system can be converged in different states by setting different energy functions.

First, the problem is mapped onto the CNN. The problem to be mapped can be represented in a commutator matrix. For a case of $n$ cities, the travel route should be represented by an $n \times n$ matrix composed of $n^{2}$ neurons.
Each row and column in the commutator matrix had only a single element, 1 , and all the other elements, 0 . Such a matrix can help us uniquely identify the shortest travel route. To have the lowest energy point in the network correspond to the shortest travel route, it is necessary to delicately construct an energy function. According to system (1), an energy function was constructed to solve the traveling salesman problem. Compared with the Hopfield neural network, the 
chaotic neural network eliminates some shortcomings, such as a lower calculation speed, a troublesome parameter setting, a larger possibility of landing in an invalid solution, and a higher difficulty in identifying the optimal solution. The expression of the energy function is given in formula (5), and the change in the energy function is shown in Figure 7. From the analysis in Figure 8, when the path optimization results are obtained, the final state of the energy function is close to 0 .

$$
E=0.5\left(x_{1} \sum_{i=1}^{n} \sum_{j=1}^{n} V_{i j}+x_{2} \sum_{j=1}^{n} \sum_{i=1}^{n} V_{i j}+x_{3} \sum_{i=1}^{n} \sum_{j=1}^{n} \mathrm{~d}_{i j} V_{i j}\right) .
$$

28 cities are set in this paper. Based on four optimization exercises, we obtain the findings: the optimal energy function is 1.5193, the initial route length was 13.1544, and the shortest route length was 5.3188. The simulation results show that the $\mathrm{CNN}$ herein can solve the traveling salesman problem very well. The results are shown in Figure 9.

\section{Conclusion}

In this paper, we put forward a new chaotic neural network model and a resulting $\mathrm{CNN}$ model. The $\mathrm{CNN}$ has rich chaotic dynamic behaviors and can generate multiscroll hidden chaotic attractors. Then, we study the dynamic behaviors, including bifurcation behaviors, Lyapunov exponent, Poincaré section, and the basins of attraction, and get knowledge of related characteristics of the basins of attraction. Furthermore, we realize the CNN through FPGA. The experiment proved that theoretical analysis and the FPGA realization led to consistent conclusions. Finally, we constructed an energy function to optimize the calculation based on the CNN, providing a new approach to solving the TSP problem. Since chaotic system and chaotic neural network have been widely used in image encryption [36-40] and secure communication [41-44], the application of these two aspects will be the focus of our future research.

\section{Data Availability}

All data used to support the findings of this study are available from the corresponding author upon request.

\section{Conflicts of Interest}

The authors declare that they have no conflicts of interest.

\section{Acknowledgments}

This research was supported in part by the National Key R\&D Program of China for International S\&T Cooperation Project (2019YFE0118700), the National Natural Science Foundation of China (61973110 and 61561022), Hunan Young Talents Science and Technology Innovation Project (2020RC3048), Natural Science Foundation of Hunan Province (2020JJ4315 and 2019JJ50648), and Education
Department of Hunan Province Outstanding Youth Project (20B216).

\section{References}

[1] W. S. McCulloch and W. H. Pitts, "A logical calculus of calculus of ideas immanent in nervous activity," The Bulletin of Mathematical Biophysics, vol. 5, pp. 115-133, 1942.

[2] P. H. Thike, Z. Zhao, P. Liu, F. Bao, and Y. Jin, "An early stopping-based artificial neural network model for atmospheric corrosion prediction of carbon steel," Computers, Materials \& Continua, vol. 65, pp. 2091-2109, 2020.

[3] B. Oh, H. Song, J. Kim, C. Park, and Y. Kim, "Predicting concentration of PM10 using optimal parameters of deep neural network," Intelligent Automation \& Soft Computing, vol. 25, pp. 343-350, 2019.

[4] V. Kůrková and M. Sanguineti, "Probabilistic lower bounds for approximation by shallow perceptron networks," Neural Networks: The Official Journal of the International Neural Network Society, vol. 91, pp. 34-41, 2017.

[5] A. Maamar and K. Benahmed, "A hybrid model for anomalies detection in ami system combining k-means clustering and deep neural network," Computers, Materials \& Continua, vol. 60, no. 1, pp. 15-39, 2019.

[6] X. Chen, Z. Wan, and J. Wang, "A study of unmanned path planning based on a double-twin rbm-bp deep neural network," Intelligent Automation \& Soft Computing, vol. 26, no. 4, pp. 1531-1548, 2020.

[7] X. Zhao, S. Chen, L. Zhou, and Y. Chen, "Sound source localization based on srp-phat spatial spectrum and deep neural network," Computers, Materials \& Continua, vol. 64, no. 1, pp. 253-271, 2020.

[8] C. Hung W, W. L. Mao, and H. Y. Huang, "Modified PSO algorithm on recurrent fuzzy neural network for system identification," Intelligent Automation \& Soft Computing, vol. 25, no. 2, pp. 329-341, 2019.

[9] C. Anitescu, E. Atroshchenko, N. Alajlan, and T. Rabczuk, "Artificial neural network methods for the solution of second order boundary value problems," Computers, Materials \& Continua, vol. 59, no. 1, pp. 345-359, 2019.

[10] F. Yu, L. Liu, L. Liu, L. Liu, and L. Liu, "A robust and fixed-time zeroing neural dynamics for computing time-variant nonlinear equation using a novel nonlinear activation function," Neurocomputing, vol. 350, pp. 108-116, 2019.

[11] E. Tlelo-Cuautle, J. Daniel Díaz-Muñoz, A. Maritza GonzálezZapata et al., "Chaotic Image Encryption Using Hopfield and Hindmarsh-Rose Neurons Implemented on FPGA," Sensors, vol. 20, p. 1326, 2020.

[12] A. Silva-Juárez, E. Tlelo-Cuautle, and Y. Gerardo, "Optimization of the Kaplan-Yorke dimension in fractional-order chaotic oscillators by metaheuristics," Applied Mathematics and Computation, vol. 394, 2021.

[13] M. Long and Y. Zeng, "Detecting Iris liveness with batch normalized convolutional neural network," Computers, $\mathrm{Ma}$ terials \& Continua, vol. 58, no. 2, pp. 493-504, 2019.

[14] C. Xiu, R. Zhou, and Y. Liu, "New chaotic memristive cellular neural network and its application in secure communication system," Chaos, Solitons \& Fractals, vol. 141, Article ID 110316, 2020.

[15] C. Jiang and Q. Chen, "Construction of blind restoration model for super-resolution image based on chaotic neural network," Chaos, Solitons \& Fractals, vol. 131, Article ID 109498, 2020. 
[16] T. Chen, L. Wang, and S. Duan, "Implementation of circuit for reconfigurable memristive chaotic neural network and its application in associative memory," Neurocomputing, vol. 380 , pp. $36-42,2020$.

[17] H.-H. Lian, S.-P. Xiao, Z. Wang, X.-H. Zhang, and H.-Q. Xiao, "Further results on sampled-data synchronization control for chaotic neural networks with actuator saturation," Neurocomputing, vol. 346, pp. 30-37, 2019.

[18] K. Guan, "Global power-rate synchronization of chaotic neural networks with proportional delay via impulsive control," Neurocomputing, vol. 283, pp. 256-265, 2018.

[19] Y. Zhao, X. Li, and P. Duan, "Observer-based sliding mode control for synchronization of delayed chaotic neural networks with unknown disturbance," Neural Networks, vol. 117, pp. 268-273, 2019.

[20] A. R. Bevi, S. Tumu, and N. V. Prasad, "Design and investigation of a chaotic neural network architecture for cryptographic applications," Computers \& Electrical Engineering, vol. 72, pp. 179-190, 2018.

[21] H. Lin, C. Wang, Y. Tan, and Y. Tan, "Hidden extreme multistability with hyperchaos and transient chaos in a Hopfield neural network affected by electromagnetic radiation," Nonlinear Dynamics, vol. 99, no. 3, pp. 2369-2386, 2020.

[22] W. Yao, C. Wang, Y. Sun, C. Zhou, and H. Lin, "Exponential multistability of memristive Cohen-Grossberg neural networks with stochastic parameter perturbations," Applied Mathematics and Computation, vol. 386, Article ID 125483, 2020.

[23] L. Cui, M. Lu, Q. Ou, H. Duan, and W. Luo, "Analysis and circuit implementation of fractional order multi-wing hidden attractors," Chaos, Solitons \& Fractals, vol. 138, Article ID 109894, 2020.

[24] Li Cui, W. Luo, and Q. Ou, "Analysis and implementation of new fractional-order multi-scroll hidden attractors," Chinese Physics B, vol. 30, no. 2, Article ID 020501, 2021.

[25] S. Camargo, R. L. Viana, and C. Anteneodo, "Intermingled basins in coupled Lorenz systems," Physical Review E, vol. 85, Article ID 036207, 2012.

[26] X. Dong and Y. Cai, "A novel genetic algorithm for large scale colored balanced traveling salesman problem," Future Generation Computer Systems, vol. 95, pp. 727-742, 2019.

[27] X. Jia, L. Pei, and R.-z. Zhu, "Application of a genetic algorithm with random crossover and dynamic mutation on the travelling salesman problem," Procedia Computer Science, vol. 131, pp. 937-945, 2018.

[28] A. El-Shamir Ezugwu, A. Oluyinka Adewumi, and M. Eduard Frîncu, "Simulated annealing based symbiotic organisms search optimization algorithm for traveling salesman problem," Expert Systems With Applications, vol. 77, pp. 189-210, 2017.

[29] I. Küçükoglu, R. Dewil, and D. Cattrysse, "Hybrid simulated annealing and tabu search method for the electric travelling salesman problem with time windows and mixed charging rates," Expert Systems With Applications, vol. 134, pp. 279$303,2019$.

[30] S. Chowdhury, M. Marufuzzaman, H. Tunc, L. Bian, and W. Bullington, "A modified Ant Colony Optimization algorithm to solve a dynamic traveling salesman problem: a case study with drones for wildlife surveillance," Journal of Computational Design and Engineering, vol. 6, no. 3, pp. 368-386, 2019.

[31] L. Chen, H.-Y. Sun, and S. Wang, "A parallel ant colony algorithm on massively parallel processors and its convergence analysis for the travelling salesman problem," Information Sciences, vol. 199, pp. 31-42, 2012.

[32] Y. Lin, Z. Bian, and X. Liu, "Developing a dynamic neighborhood structure for an adaptive hybrid simulated annealing - tabu search algorithm to solve the symmetrical traveling salesman problem," Applied Soft Computing, vol. 49, pp. 937-952, 2016.

[33] J. Brecklinghaus and S. Hougardy, "The approximation ratio of the greedy algorithm for the metric traveling salesman problem," Operations Research Letters, vol. 43, no. 3, pp. 259-261, 2015.

[34] X. Geng, Z. Chen, W. Yang, D. Shi, and K. Zhao, "Solving the traveling salesman problem based on an adaptive simulated annealing algorithm with greedy search," Applied Soft Computing, vol. 11, no. 4, pp. 3680-3689, 2011.

[35] Y. Hu, Z. Zhang, Y. Yao, X. Huyan, X. Zhou, and W. S. Lee, “A bidirectional graph neural network for traveling salesman problems on arbitrary symmetric graphs," Engineering Applications of Artificial Intelligence, vol. 97, Article ID 104061, 2021.

[36] F. Yu, S. Qian, X. Chen et al., "Chaos-based engineering applications with a 6D memristive multistable hyperchaotic system and a 2D SF-SIMM hyperchaotic map," Complexity, vol. 2021, Article ID 6683284, 21 pages, 2021.

[37] B. Lu, F. Liu, X. Ge, and Z. Li, "Cryptanalysis and improvement of a chaotic map-control-based and the plain image-related cryptosystem," Computers, Materials \& Continua, vol. 61, no. 2, pp. 687-699, 2019.

[38] Xi Chen, S. Qian, Yu Fei et al., "Pseudorandom number generator based on three kinds of four-wing memristive hyperchaotic system and its application in image encryption," Complexity, vol. 2020, Article ID 8274685, 17 pages, 2020.

[39] X.-Y. Wang and Z.-M. Li, "A color image encryption algorithm based on Hopfield chaotic neural network," Optics and Lasers in Engineering, vol. 115, pp. 107-118, 2019.

[40] J. Liu, J. Li, J. Cheng et al., "A novel robust watermarking algorithm for encrypted medical image based on dtcwt-dct and chaotic map," Computers, Materials \& Continua, vol. 61, no. 2, pp. 889-910, 2019.

[41] W. He, T. Luo, Y. Tang et al., "Secure communication based on quantized synchronization of chaotic neural networks under an event-triggered strategy," IEEE Transactions on Neural Networks and Learning Systems, vol. 31, no. 9, pp. 3334-3345, 2019.

[42] G. Xu, J. Xu, C. Xiu, F. Liu, and Y. Zang, "Secure communication based on the synchronous control of hysteretic chaotic neuron," Neurocomputing, vol. 227, pp. 108-112, 2017.

[43] L. Zhou, F. Tan, and F. Yu, "A robust synchronization-based chaotic secure communication scheme with double-layered and multiple hybrid networks," IEEE Systems Journal, vol. 14, no. 2, pp. 2508-2519, 2019.

[44] J. Liu and R. Xu, "Adaptive synchronisation of memristorbased neural networks with leakage delays and applications in chaotic masking secure communication," International Journal of Systems Science, vol. 49, no. 6, pp. 1300-1315, 2018. 